\title{
ORIGINAL
}

\section{EVALUACIÓN DE UNA INTERVENCIÓN PARA DEJAR DE FUMAR EN PERSONAS CON ENFERMEDAD MENTAL}

\section{Luis Gutiérrez Bardeci (1), Luis Otero (1), Mónica del Amo (1) y F Javier Ayesta (2).}

(1) Centro de Salud Puertochico. Servicio Cántabro de Salud.

(2) Departamento de Fisiología y Farmacología, Universidad de Cantabria.

El presente trabajo no ha recibido ninguna subvención o ayuda.

Los autores no tienen conflictos de interés relacionados con la materia del trabajo.

\section{RESUMEN}

Fundamentos: Una alta proporción de quienes solicitan ayuda para dejar de fumar padecen trastornos psiquiátricos. El objetivo del trabajo es conocer si las intervenciones grupales recomendadas para la atención de personas fumadoras son efectivas en pacientes con antecedentes de patología psiquiátrica y si ello supone un factor pronóstico negativo.

Métodos: Estudio descriptivo longitudinal con un año de seguimiento. Se evaluaron los grupos de deshabituación realizados en atención primaria durante el período 2006-11, incluyendo a 267 fumadores, 97 de ellos con antecedentes personales de patología psiquiátrica. Se realizaron cinco sesiones presenciales de 90 minutos (hasta $6^{\mathrm{a}}$ semana abstinencia) y seguimiento telefónico posterior de 12 meses. La abstinencia fue comprobada por cooximetría en las sesiones presenciales y auto-declarada posteriormente. Análisis estadístico: descripción de variables cualitativas y cuantitativas, análisis comparativo de las mismas entre los dos grupos y regresión logística binaria según cesación.

Resultados: La tasa de abstinencia continua declarada al año fue $39,7 \%$, en mujeres del $43,1 \%$ y en hombres $35,1 \%$; $: 0,18$ ). Las personas fumadoras sin historia psiquiátrica presentaron tasas de abstinencia del $42,9 \%$ y las que sí la tenían del 34,0\% (p: 0,16). No hubo interacción entre las variables sexo y patología psiquiátrica. Quienes presentaban antecedentes psiquiátricos recayeron en las 6 primeras semanas tras la abstinencia en el 23,7\% y quienes no los tenían:10,6\% [(p:0,019; OR:1,90 [LC95\%:1,08-3,36)]; a partir de entonces las tasas de recaída fueron similares recayendo el $44,1 \%$ y un $42,5 \%$, respectivamente $(\mathrm{p}>0,8)$.

Conclusiones: Las personas con patología psiquiátrica asociada o previa parecen encontrar más dificultades para dejar de fumar pero la intervención es efectiva en ambos grupos.

Palabras clave: Tabaquismo. Atención Primaria de salud. Trastornos mentales. Terapia en grupo.

Correspondencia

Luis Gutiérrez Bardeci

CS Puertochico

c/ Tetuán 54

39004 Santander

luis.gutierrez@scsalud.es

\section{ABSTRACT \\ Evaluation of the intervention to Quit Smoking in People with Mentall Illness}

Background: A high proportion of those seeking help to quit smoking have psychiatric disorders. The aim of this work is to analyze the effectiveness of those group smoking cessation programs who are usually employed in Primary Care and to compare their results to those obtained with non psychiatric patients.

Methods: A descriptive longitudinal study with a one-year follow-up that evaluated smoking cessation groups conducted in a Primary Care setting between 2006 and 2011. Nineteen groups were carried out and 267 smokers were included; $36.3 \%$ had been diagnosed with a psychiatric disorder. The program consisted of five 90 minutes sessions (up to six weeks after abstinence) with telephonic follow-up for a year. Abstinence was verified by cooximetry at the end of the program and by self-declaration afterwards. Quantitative and qualitative variables were described, and the association between variables analyzed through binary logistic regression.

Results: Self-declared continuous abstinence rate at 12 months of was $39.7 \%$, being slightly higher, not in a non significant way, in women $(43.1 \%$ vs. $35.1 \%$; p: 0.18$)$. Smokers with no history of psychiatric illness showed better abstinence rates, although without significant differences $(42.9 \%$ vs. $34.0 \%$; p: 0.16$)$. There was no significant interaction between sex and psychiatric pathology. Those with a psychiatric disorder relapsed in a significantly higher rate during the first six weeks after abstinence [23.7\% vs. $10.6 \%$, p: 0.019 ; OR:1.90(1.08-3.36)]; relapse rates were similar afterwards $(44,1 \%$ vs. $42,5 \% ; \mathrm{p}>0,8)$.

Conclusions: This group intervention program for smoking cessation is effective. Although smokers with a present or previous history of psychiatric disorder may find abstinence harder to attain, they benefit from the intervention.

Keywords: Tobacco. Primary health care. Mental disorders. Psychotherapy, group. 


\section{INTRODUCCIÓN}

Existe una clara asociación epidemiológica entre consumo de tabaco y trastornos mentales en la población general: quienes padecen un trastorno mental tienen el doble de posibilidades de ser fumadores que quienes no lo padecen $^{1-4}$. Se estima que en la actualidad el $30 \%$ de quienes fuman y el $45 \%$ de quienes piden ayuda para dejar de fumar presentan algún trastorno mental ${ }^{5,6}$.

Las personas fumadoras con patología psiquiátrica asociada presentan mayores dificultades para abandonar su consumo de tabaco, por lo que pueden precisar intervenciones más intensivas $\mathrm{y} / \mathrm{o}$ más prolongadas ${ }^{7,8}$, motivo por el cual la presencia de patología psiquiátrica es un criterio de posible derivación a unidades más especializadas ${ }^{9}$. Se ha postulado que esta demanda se incrementará a medida que disminuya la prevalencia de fumadores de la población general, situación en la que se hace más evidente la asociación entre dependencia nicotínica y patología psiquiátrica ${ }^{1,3,10}$.

La mayoría de quienes solicitan ayuda profesional para la cesación acuden a atención primaria (AP). Parte de estos presentan historia de trastornos psiquiátricos.

Por estos motivos es importante conocer si los programas que se realizan habitualmente en AP son también efectivos en estos pacientes en los que, al grave riesgo del consumo de tabaco, se añaden los problemas derivados de su patología psiquiátrica.

El objetivo de la investigación es conocer si las intervenciones grupales que se recomiendan para la atención de personas fumadoras en AP son efectivas en los pacientes con patología psiquiátrica y evaluar si la presencia de antecedentes psiquiátricos o de enfermedad psiquiátrica supone un factor pronóstico negativo, así como conocer si variables demográficas o del propio consumo de tabaco se pueden relacionar con la abstinencia en el hábito de fumar.

\section{SUJETOS Y MÉTODOS}

En el estudio se incluyó a todas las personas fumadoras que solicitaron soporte para dejar de fumar y recibieron tratamiento multicomponente en grupo durante el período abril 2006-junio 2011 en el Centro de Salud (CS) Puertochico de Santander. Este CS es responsable de la atención sanitaria de 22.000 personas. Del desarrollo de los grupos se encargaron dos médicos y una enfermera.

La captación de personas fumadoras interesadas comenzó dos meses antes del inicio de cada uno de los grupos, labor en la que participaron de forma activa todos los profesionales de medicina y enfermería del CS. El programa para dejar de fumar se publicitó mediante carteles informativos.

Para participar se establecieron como criterios de inclusión haber solicitado ayuda para dejar de fumar, preferir el formato grupal y ser mayor de edad. Se excluyó a las personas fumadoras con dificultad de integración en el grupo (por padecer déficit sensoriales auditivos o presentar dificultades con el idioma), con trastorno psicótico, con otros trastornos adictivos o con cualquier patología psiquiátrica no estabilizada.

La presencia de trastorno psiquiátrico no psicótico estable o el antecedente de haberlo padecido no fueron motivos de exclusión. A las personas con trastornos psiquiátricos que querían dejar de fumar y no se consideró indicado incluirlas en los grupos se les propuso el formato individual o la derivación a la unidad especializada de la comunidad autónoma.

Una vez seleccionados, los participantes fueron citados en la consulta programada para la entrevista basal previa en la que se realizaba la historia clínica de tabaquismo, que incluía la medición del monóxido de carbono (CO) en aire espirado. Esta consulta servía para tener un primer contacto entre 
terapeuta y paciente, individualizar la intervención, entregar el calendario de las sesiones y concretar un tratamiento farmacológico, según las pautas eficaces establecidas por la evidencia ${ }^{8,11}$. A todos los participantes se les propuso tratamiento farmacológico con vareniclina, bupropión o parches de nicotina, acompañados de formas de liberación puntual de tratamiento sustitutivo de nicotina (TSN) en forma de chicles o comprimidos. La indicación de cada principio activo concreto vino determinada por la ausencia de contraindicaciones y la preferencia de cada participante, dado que entre los fármacos de primera línea no existe evidencia que sirva para indicar un fármaco u otro ${ }^{8}$.

La anamnesis y la revisión de la historia clínica informatizada (OMI-AP) permitieron conocer qué participantess habían padecido o padecían enfermedad psiquiátrica.

Tras la entrevista basal, las personas fumadoras acudieron a cinco sesiones presenciales de unos 90 minutos cada una, dos de preparación antes de dejar de fumar y tres de mantenimiento de la abstinencia, con una periodicidad semanal las tres primeras, 15 días después la cuarta y tres semanas después la quinta. Todas las sesiones se integraron en el horario habitual del centro, el mismo día de la semana y a la misma hora.

Una vez finalizadas las 5 sesiones se realizaron seguimientos telefónicos a los dos, tres, seis meses y al año del cese del consumo de tabaco. Este tipo de intervención refuerza la abstinencia, sirve para controlar el tratamiento farmacológico y es posible que reduzca la probabilidad de recaídas ${ }^{8,12}$.

Este protocolo de intervención se ajusta a las recomendaciones formuladas por el Documento Técnico de consenso sobre la atención sanitaria del tabaquismo en España ${ }^{9}$ y por la Consejería de Sanidad de Cantabria $^{12}$.
La abstinencia continua se determinó mediante cooximetría y autodeclaración en las sesiones presenciales y al final de las mismas (6 semanas de abstinencia) y posteriormente mediante autodeclaración. Se consideró no abstinente al paciente con un valor de $\mathrm{CO}$ en el aire espirado mayor de 6 partes por millón (ppm) o por la imposibilidad de contacto con el paciente tras tres llamadas telefónicas.

Además del sexo y edad se analizaron las siguiente variables: 1) relacionadas con el consumo de tabaco: edad de inicio, número de cigarrillos/día, cooximetría al inicio, test de Fagerström (FTND), número de intentos previos; 2) relacionadas con el tratamiento: tratamiento farmacológico empleado, asistencia a las sesiones; 3) variables clínicas: peso inicial y ganancia ponderal, tensión arterial sistólica (TAS), tensión arterial diastólica (TAD), enfermedad psiquiátrica estable o antecedentes de haberla padecido.

Análisis estadístico. Se realizó un análisis descriptivo presentándose las variables cualitativas como proporciones y de las variables cuantitativas su media y error estándar. La asociación entre variables se estudió mediante el modelo univariante empleando la prueba de la $t$ de Student en las variables cuantitativas y la prueba de la chi $^{2}$ en las cualitativas. También se realizó análisis de regresión logística binaria (variable dependiente: cesación a los 12 meses) para descartar variables de confusión. Todas las pruebas se realizaron a dos colas y el nivel de significación se estableció en 0,05 .

\section{RESULTADOS}

En el período analizado (abril 2006-junio 2011) se realizaron 19 grupos, en los que hubo un total de 267 participantes (153 mujeres y 114 varones). 27 personas realizaron la entrevista basal pero no iniciaron el tratamiento grupal: 3 de ellas porque se consideró más adecuada la intervención en for- 
mato individual, 6 porque fueron derivadas a la unidad especializada y el resto porque decidieron no iniciar el programa tras el período de espera. No fueron incluidas en el tamaño de la muestra. Del total de 294 personas que solicitaron soporte para dejar de fumar 109 (37\%) tenían reflejada en su historia clínica episodios psiquiátricos (trastornos psicóticos, de ansiedad o del estado de ánimo) y/o tratamiento crónico para los mismos. De ellas, 12 no comenzaron el tratamiento.

Características de la muestra (tabla 1). De las personas que empezaron los grupos de tratamiento $97(36,3 \%)$ presentaban en su historia clínica un trastorno psiquiátrico actual (depresivo o ansioso). Por sexos lo presentaban $75(49,0 \%)$ de las 153 mujeres y $22(19,3 \%)$ de los 114 hombres $(\mathrm{p}<0,0001)$.

Los varones presentaron un nivel de $\mathrm{CO}$ en aire espirado de 23,0 ppm y las mujeres de 19,9 ppm (p: 0,009), no apreciándose diferencias en las puntuaciones del test de Fagerström (FTND) ni en la edad de inicio en el consumo, mayor consumo de cigarrillos (2,2 cig/d más, p:0,07) y de intentos de abandono (0,37 intentos más; $p: 0,08)$. Tam- bién se apreciaron diferencias por sexos en las cifras de tensión arterial, que fueron mayores en los varones $(8,2 \mathrm{mmHg}$ en la sistólica y 5,7 en la diastólica) y en el peso al inicio del tratamiento (tabla 1).

La ganancia ponderal durante las 6 semanas de tratamiento fue similar entre las personas con historia psiquiátrica y las que no la tenían $(0,1$ vs $1,2 \mathrm{~kg}$ en mujeres y 6,6 vs. 4,6 en varones ( $p>0,1$ en ambos casos).

El análisis de las variables según la existencia o no antecedentes psiquiátricos (tabla 2) mostró la inexistencia de diferencias en las variables analizadas en los varones. En el caso de las mujeres se observó que quienes presentaban antecedentes psiquiátricos fumaban 3 cigarrillos más al día y puntuaban 0,7 puntos más en el FTND.

De las 267 personas que iniciaron el tratamiento en los grupos, $40(15,0 \%)$ no llegaron al día $\mathrm{D}$ en el que se debe dejar de fumar. $41(15,3 \%)$ recayeron antes de la $6^{\mathrm{a}}$ semana tras el día $\mathrm{D}$ (fecha de la $5^{\mathrm{a}}$ y última sesión presencial en la que se realiza una cooximetría de validación). Las restantes 186 $(69,7 \%)$ que se mantuvieron abstinentes hasta esa fecha acudieron a 4,64 sesiones de

Tabla 1

\section{Características de la muestra}

\begin{tabular}{|l|c|c|c|}
\hline \multicolumn{1}{|c|}{ Variables } & $\begin{array}{c}\text { Mujeres (n: 153) } \\
\text { (Media } \pm \text { Error Estándar) }\end{array}$ & $\begin{array}{c}\text { Varones (n:114) } \\
\text { (Media } \pm \text { Error Estándar) }\end{array}$ & $\mathrm{p}$ \\
\hline Antecedentes psiq. & $75 / 153(49,0 \%)$ & $22 / 114(19,3 \%)$ & $<0,0001$ \\
\hline Edad & $47,2 \pm 1,0$ & $48,3 \pm 1,1$ & 0,464 \\
\hline Peso inicial & $65,1 \pm 0,9$ & $82,4 \pm 1,4$ & $<0,0001$ \\
\hline TAS $^{*}$ & $121,2 \pm 1,5$ & $129,4 \pm 1,5$ & 0,0002 \\
\hline TAD $^{\dagger}$ & $73,4 \pm 0,9$ & $79,2 \pm 0,9$ & $<0,0001$ \\
\hline Cig/d & $21,3 \pm 0,7$ & $23,5 \pm 1,0$ & 0,070 \\
\hline CO & $19,9 \pm 0,7$ & $23,0 \pm 0,9$ & 0,009 \\
\hline FTND & $5,5 \pm 0,2$ & $5,6 \pm 0,2$ & 0,620 \\
\hline Edad de inicio & $16,8 \pm 0,4$ & $16,3 \pm 0,4$ & 0,360 \\
\hline $\mathrm{N}^{\mathbf{0}}$ intentos & $1,61 \pm 0,14$ & $1,98 \pm 0,16$ & 0,081 \\
\hline
\end{tabular}

*TAS : Tensión arterial sistólica. †TAD: Tensión arterial diastólica. ${ }^{\star}$ FNTD: Test de Fagerström. 
Tabla 2

Diferencias en las variables según sexo y existencia de historia psiquiátrica

\begin{tabular}{|l|c|c|c|c|c|c|}
\hline \multirow{2}{*}{} & \multicolumn{3}{|c|}{$\begin{array}{c}\text { Mujeres } \\
\text { (Media } \pm \text { Error Estándar) }\end{array}$} & \multicolumn{3}{c|}{$\begin{array}{c}\text { Hombres } \\
\text { (Media } \pm \text { Error Estándar) }\end{array}$} \\
\cline { 2 - 7 } & Psiq (75) & No psiq (78) & $\mathrm{p}$ & Psiq (22) & No psiq (92) & $\mathrm{p}$ \\
\hline Edad & $48,0 \pm 1,2$ & $46,5 \pm 1,5$ & 0,43 & $49,7 \pm 3,1$ & $48,0 \pm 1,2$ & 0,54 \\
\hline Peso inicial & $65,1 \pm 1,4$ & $65,0 \pm 1,2$ & 0,98 & $80,3 \pm 3,2$ & $82,9 \pm 1,5$ & 0,45 \\
\hline TAS $^{*}$ & $121,8 \pm 1,9$ & $120,6 \pm 2,4$ & 0,68 & $130,6 \pm 3,6$ & $129,1 \pm 1,7$ & 0,69 \\
\hline TAD $^{\dagger}$ & $73,6 \pm 1,5$ & $73,3 \pm 1,1$ & 0,29 & $77,4 \pm 2,1$ & $79,6 \pm 1,0$ & 0,35 \\
\hline Cig/d & $22,8 \pm 1,0$ & $19,8 \pm 1,1$ & $0,042^{*}$ & $23,0 \pm 2,2$ & $23,7 \pm 1,2$ & 0,78 \\
\hline CO & $19,6 \pm 1,0$ & $20,2 \pm 1,1$ & 0,70 & $22,4 \pm 1,9$ & $23,1 \pm 1,1$ & 0,75 \\
\hline FTND & $5,8 \pm 0,2$ & $5,1 \pm 0,3$ & $0,039 *$ & $6,3 \pm 0,5$ & $5,5 \pm 0,2$ & 0,12 \\
\hline Edad de inicio & $16,4 \pm 0,4$ & $17,3 \pm 0,6$ & 0,21 & $15,4 \pm 0,6$ & $16,5 \pm 0,5$ & 0,27 \\
\hline$N^{\circ}$ intentos & $1,82 \pm 0,22$ & $1,40 \pm 0,17$ & 0,13 & $1,73 \pm 0,32$ & $2,04 \pm 0,19$ & 0,45 \\
\hline
\end{tabular}

*TAS : Tensión arterial sistólica. †TAD: Tensión arterial diastólica. ${ }^{\star F N T D: ~ T e s t ~ d e ~ F a g e r s t r o ̈ m . ~}$

promedio, no apreciándose diferencias estadísticamente significativas entre quienes acabaron recayendo posteriormente $(4,59)$ y quienes no $(4,69)$.

La tasa global de abstinencia cayó del $69,7 \%$ a las 6 semanas al $51,3 \%$ al cabo de 6 meses y al 39,7\% al cabo de un año (abstinencia declarada en ambos casos). Aunque la tasa de abstinencia fue ligeramente superior en mujeres que en varones $(43,1 \%$ vs. $35,1)$ las diferencias no fueron estadística- mente significativas (p: 0,150 en el análisis multivariante descartando factores de confusión) (tabla 3). La tasa de abstinencia entre las 31 personas que utilizaron parches de nicotina fue del $22,6 \%$, significativamente menor (p:0,034) que la de quienes utilizaron vareniclina $(38$ de $76,50,0 \%)$ y bupropion (49 de 115, 42,6\%), entre los que no hubo diferencias estadísticamente significativas. Rehusaron utilizar fármacos 11 $(4,1 \%)$ sujetos.

Tabla 3

Análisis multivariante controlado por posibles factores de confusión de la influencia de las variables analizadas en la tasa de recaída a los 12 meses, con todas las variables (izquierda) y eliminando las retrospectivamente (backwards) las menos significativas hasta conseguir el mejor modelo (derecha)

\begin{tabular}{|l|c|c|c|c|c|c|}
\hline & $\operatorname{Exp}(\mathrm{B})$ & $\mathrm{IC} 95,0 \%$ & $\mathrm{p}$ & $\operatorname{Exp}(\mathrm{B})$ & $\mathrm{IC} 95,0 \%$ & $\mathrm{p}$ \\
\hline Pat. Psiquiátrica* & 1,61 & $0,91-2,94$ & 0,098 & 1,65 & $0,94-2,89$ & 0,078 \\
\hline Sexo** & 1,49 & $0,86-2,59$ & 0,150 & 1,52 & $0,88-2,62$ & 0,131 \\
\hline $\mathrm{N}^{\circ}$ intentos & 1,14 & $0,97-1,34$ & 0,111 & 1,14 & $0,97-1,34$ & 0,099 \\
\hline FTND*** & 1,04 & $0,90-1,21$ & 0,561 & & & \\
\hline Edad inicio & 1,04 & $0,92-1,05$ & 0,621 & & & \\
\hline $\mathrm{N}^{\text {o }}$ cigarrillos & 1,01 & $0,98-1,05$ & 0,441 & 1,02 & $0,99-1,05$ & 0,107 \\
\hline Niveles CO & 1,01 & $0,98-1,04$ & 0,724 & & & \\
\hline Edad actual & 1,00 & $0,98-1,02$ & 0,958 & & & \\
\hline
\end{tabular}

*Categoría de referencia: presencia de patología psiquiátrica; **Categoría de referencia: varones; ***FNTD: Test de Fagerström. 
Rehusaron utilizar fármacos 11(4,1\%) sujetos.

La figura 1 muestra la evolución de la abstinencia en los períodos evaluados según la existencia o no de historia psiquiátrica. La tasa de abstinencia a los 12 meses en las personas sin historia psiquiátrica fue del $42,9 \%$ y en las personas con antecedentes de trastornos psiquiátricos del 34,0\% (p:0,098) este valor se incrementó hasta 0,078 tras la eliminación progresiva de las variables menos significativas del modelo (tabla 3). Las tasas de abstinencia de las personas con historia psiquiátrica fueron menores en ambos sexos, aunque las diferencias no alcanzaron el nivel de significación estadística aceptado (figura 2), posiblemente por un tamaño insuficiente de la muestra.
El análisis del curso temporal de las recaídas (figura 1) mostró que:

1) no existieron diferencias entre ambos grupos $(15,5 \%$ vs. $14,7 \%, p>0,8)$ en el porcentaje de quienes no llegaron a conseguir un día de abstinencia, es decir, de quienes no llegaron al día $\mathrm{D}$.

2) las personas con historia de antecedentes psiquiátricos recayeron significativamente más en el periodo comprendido entre el día $\mathrm{D}$ y el término de las 5 sesiones (semana $6^{\mathrm{a}}$ tras el cese): $23,7 \%$ vs. $10,6 \%$ (p: 0,019 ; OR: 1,90 ; IC95\%: 1,08-3,36).

3) El 44,1\% de las personas con antecedentes psiquiátricos que llegaron abstinentes a la $6^{\mathrm{a}}$ semana (final de las sesiones) recayó entre esta fecha y los 12 meses, porcentaje que no difirió de la encontrada en la población no psi-

Figura 1

Abstinencia continua validada (6 semanas; al terminar el taller) y declarada (6 y 12 meses) según la existencia o no de historia psiquiátrica

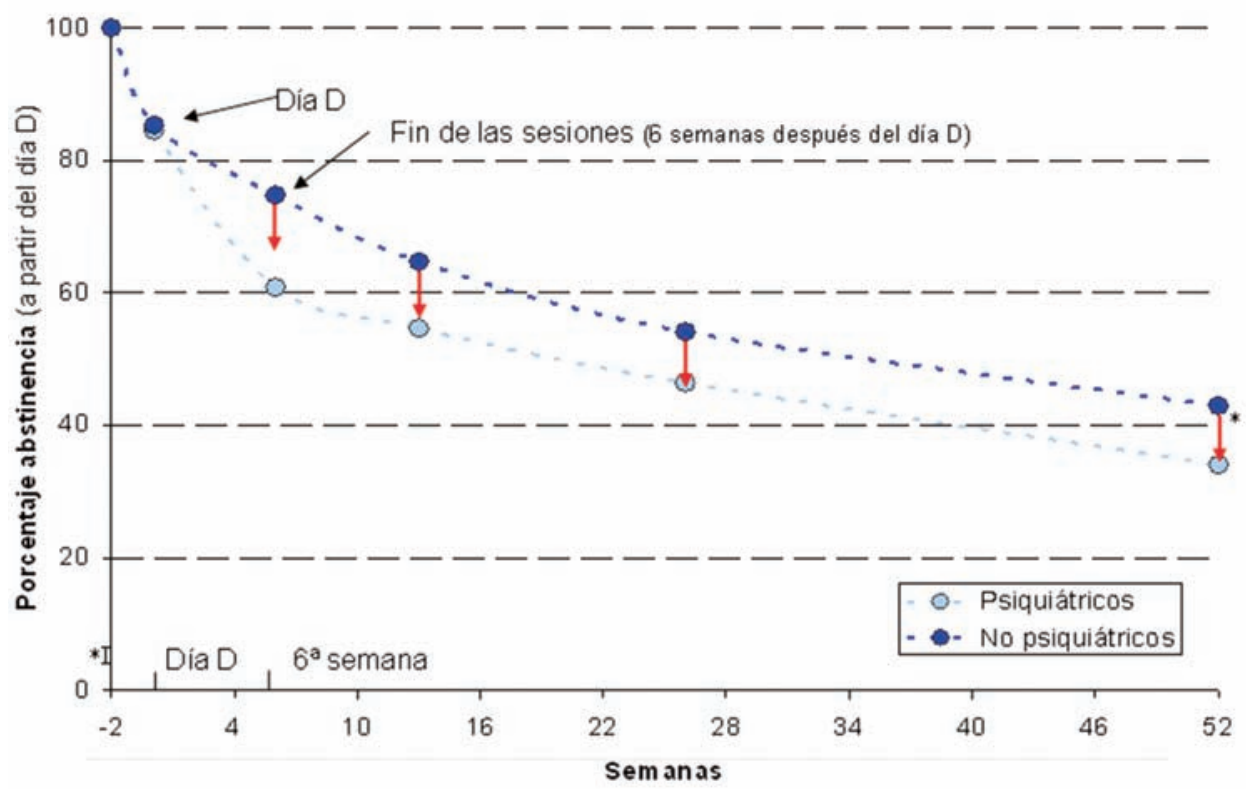


Figura 2

Tasa de abstinencia continua declarada a los 12 meses según sexo y la existencia de historia psiquiátrica

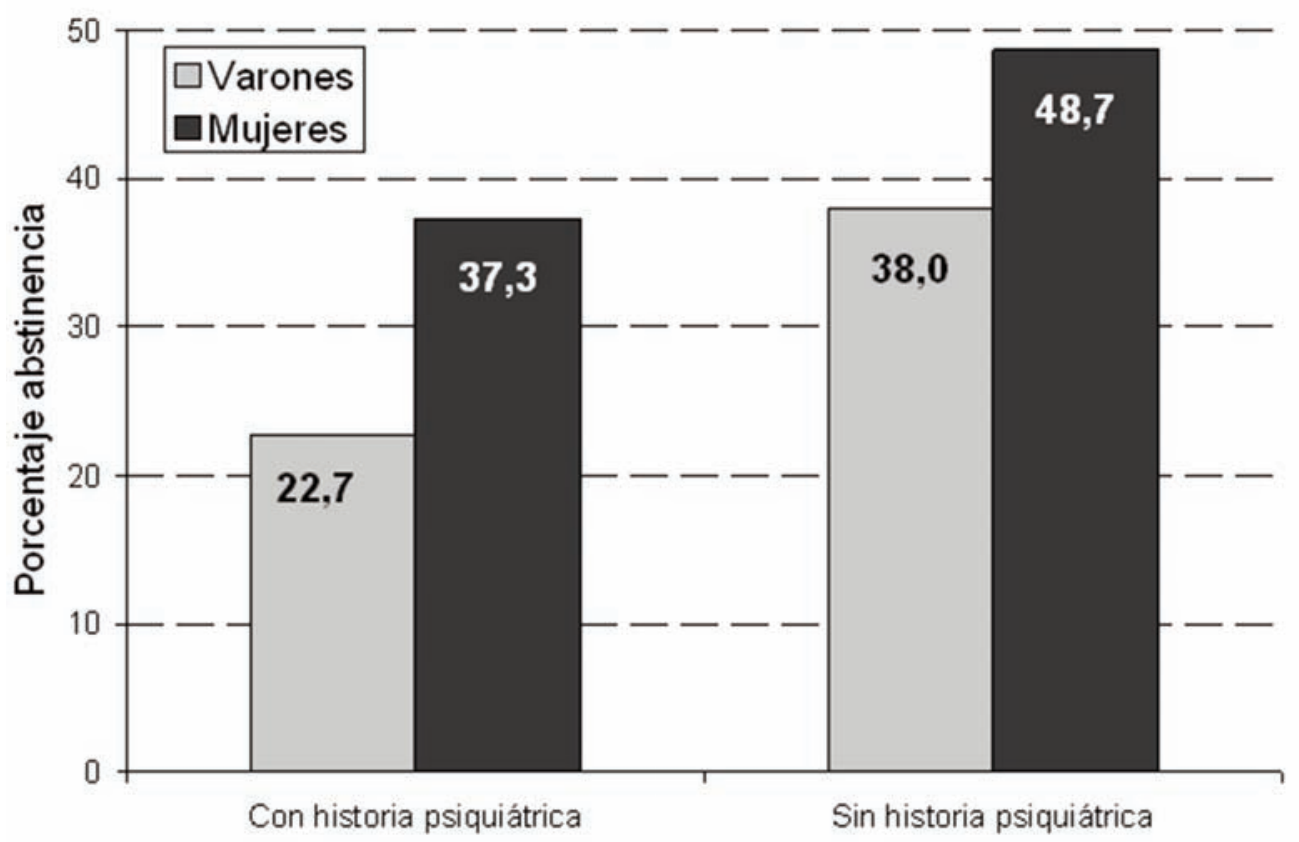

quiátrica: durante ese periodo recayó el $42,5 \%$ de quienes estaban abstinentes a la $6^{\mathrm{a}}$ semana $(\mathrm{p}>0,8)$.

\section{DISCUSIÓN}

El presente estudio muestra la efectividad de la intervención que se realiza. La abstinencia continua declarada al año fue del $40 \%$, cifra relevante en un trastorno adictivo que supone un grave factor de riesgo de enfermedad.

Un empleo más intensivo de técnicas motivacionales en las sesiones de preparación podría servir para disminuir el número de personas -uno de cada seis, aproximadamente- que, habiendo iniciado el tratamiento, no llegó al día $\mathrm{D}$, es decir, no consiguió dejar de fumar ni un día ${ }^{6}$.
El hecho de que entre el $6^{\circ}$ y el $12^{\circ}$ mes recaiga casi uno de cada cuatro participantes sugiere que es posible que al menos una parte de ellos podría beneficiarse de un seguimiento más prolongado, quizá aumentando el número de contactos oportunistas o programados, o incrementando el número de seguimientos telefónicos ${ }^{4,8}$.

Una limitación del estudio es la falta de confirmación de la abstinencia por cooximetría u otros marcadores biológicos una vez pasados los dos primeros meses. No obstante, según las directrices de la Society for Research on Nicotine and Tobacco $(\mathrm{SRNT})^{13}$, la abstinencia declarada puede considerarse válida en este tipo de estudios y la mayoría de las publicaciones que revisan la validez de la auto-declaración de abstinencia señalan que el porcentaje de falsos 
negativos es inferior al $10 \%{ }^{14}$. Por ello, se estima que la fiabilidad de la respuesta es habitualmente elevada y debe ser tenida en cuenta $^{14-16}$, máxime cuando al finalizar los grupos los resultados de la cooximetría y la auto-declaración concuerdan plenamente y se mantiene el vínculo terapéutico.

La utilización de tratamientos farmacológicos para ayudar a dejar de fumar duplica aproximadamente la efectividad de las intervenciones, sobre todo cuando se asocia TSN de modo puntual y es recomendada por todas las guías ${ }^{8,11,17}$. La alta tasa de empleo de fármacos útiles (superior al 95\%), unido al hecho de que a todos los participantes se les ofreciera TSN puntual a modo de rescate, podría explicar las mayores tasas de abstinencia a largo plazo que se obtienen en el presente trabajo, en comparación con otros que siguen programas similares ${ }^{18,19}$.

Aunque los diversos metanálisis muestran que la eficacia de la TSN en parches es algo menor en comparación con vareniclina (entre $26 \%$ y $39 \%)^{11}$, carecemos de una explicación definitiva que justifique las menores tasas -prácticamente la mitad- que se consiguen en nuestro estudio con los parches.

Aunque la diferencia no es estadísticamente significativa, la tasa de abstinencia que se consigue en mujeres es algo superior a la que se obtiene en varones, lo que se observa independientemente de que exista o no historia de enfermedad psiquiátrica. Es posible que las diferencias observadas (número de intentos, cigarrillos/día y $\mathrm{CO}$ ), descritas también en estudios previos ${ }^{20}$, puedan justificar parcialmente esta diferencia por sexos, la cual hubiera necesitado de más del doble de pacientes en la muestra para alcanzar la significación estadística.

El porcentaje de personas de la muestra que solicitaron soporte para dejar de fumar y que presentaban episodios psiquiátricos en la historia clínica (algo más de un tercio de la muestra) es similar al referido en otros estu$\operatorname{dios}^{1,4,6}$. La prevalencia de enfermedad mental en quienes en el presente estudio solicitaron ayuda para dejar de fumar es más del doble de la referida en población general en estudios españoles, la cual oscila en torno al $20 \%$ en mujeres y el $8 \%$ en varones ${ }^{21}$.

La tasa de abstinencia al año de los participantes con patología psiquiátrica o con antecedentes de haberla padecido -aun siendo inferior de manera no significativa al resto de población fumadora- fue superior al $30 \%$. Esto muestra que las personas con historia de enfermedad psiquiátrica pueden beneficiarse también de las intervenciones habituales ${ }^{22-24}$ y que los antecedentes psiquiátricos no deben ser un motivo para inhibirse en el tratamiento de quienes solicitan ayuda para dejar de fumar ${ }^{4,5}$. Aunque los datos no son estrictamente comparables, ya que existen diferencias en la selección de los participantes, son similares a los obtenidos a los 6 meses por el grupo de Baker $^{25}$, que encontró una tasa de abstinencia del 35,4\% en quienes nunca han sido diagnosticados de un trastorno psiquiátrico y de un $31,2 \%$ y $28,7 \%$ en quienes han sido alguna vez diagnosticados de padecer algún trastorno depresivo o de ansiedad, respectivamente.

Aunque las diferencias no son estadísticamente significativas, la menor tasa de abstinencia de los participantes con patología psiquiátrica podría explicarse en parte por una mayor dependencia, como indican su mayor puntuación en el FNTD y en mujeres su mayor consumo diario de cigarrillos. Es posible también que estas personas experimenten los síntomas más intensamente o que presenten menos recursos espontáneos para realizar el cambio de conducta que comporta la cesación, cuestiones ambas que podrían favorecer las recaídas ${ }^{4,10}$. Estas variables no fueron analizadas en el presente estudio.

Que el porcentaje de participantes con antecedentes psiquiátricos que llega al día $\mathrm{D}$ sea igual del resto de personas fumadoras 
sugiere que no existen en ellos déficits de motivación respecto a la iniciación del proceso terapéutico. El hecho de que los pacientes con antecedentes psiquiátricos recaigan significativamente más en las seis primeras semanas de abstinencia pero que no lo hagan posteriormente, sugiere que podrían beneficiarse de intervenciones más intensivas a lo largo de estas seis primeras semanas, período en el que parecen concentrarse sus dificultades. Esta cuestión ha sido planteada en diversos artículos ${ }^{4,7,23,24}$ y parece refrendada por nuestros resultados.

En definitiva, los resultados muestran que este modelo de intervención de deshabituación tabáquica es efectiva en el grupo estudiado tanto en personas con historia de trastorno psiquiátrico como sin ella, y que las que tienen antecedentes pueden beneficiarse de la intervención para dejar de fumar sin necesidad de ser sistemáticamente derivadas a unidades especializadas. Así mismo, estos resultados parecen confirmar la mayor dificultad que tienen estas personas para abandonar el consumo de tabaco, lo que resalta la importancia de ofertarles ayuda para ello.

\section{BIBLIOGRAFÍA}

1. Lawrence D, Mittrou F, Zubrick SR. Smoking and mental illness: results from population surveys in Australia and the United States. BMC Public Health. 2009 9:285.

2. Griesler PC, Hu MC, Schaffan C, Kandel BD. Comorbid psychiatric disorders and nicotine dependence in adolescence. Addiction. 2011; 106: 1010-1020.

3. Schroeder SA., Morris CD. Confronting a neglected epidemic: Tobacco cessation for persons with mental illness and substance abuse problems. Annu Rev Public Health. 2010; 31: 297-314.

4. Ziedonis D, Hitsman B, Beckham JC, Zvolensky M, Adler LE, Audrain-McGovern J et al. Tobacco use and cessation in psychiatric disorders: National Institute of Mental Health report. Nicotine Tob Res. 2008; 10:1691-1715.

5. Prochaska JJ. Smoking and mental illness - breaking the link. N Eng J Med. 2011; 365: 196-198.
6. Sobradiel N, García-Vicent V. Consumo de tabaco y patología psiquiátrica. Trastornos Adictivos. 2007; 9: $31-38$

7. Ayesta FJ, Rodríguez M. Bases biológicas y sociales de las dependencias: tabaco y nicotina. Santander: Manuales Piufet; 2007.

8. Fiore MC, Jaén CR, Baker TB, et al. Treating Tobacco Use and Dependence: 2008 Update. Clinical Practice Guideline. Rockville, MD: U.S. Department of Health and Human Services. Public Health Service; 2008.

9. Documento técnico de consenso sobre la atención sanitaria del tabaquismo en España. Observatorio para Prevención del Tabaquismo. Madrid: CNPT-Ministerio de Sanidad; 2008.

10. Aubin HJ, Rollema H, Svensson TH, Winterer G. Smoking, quitting, and psychiatric disease: a review. Neurosci Biobehav Rev. 2012; 36: 271-284.

11. Olano E, Minué C, Ayesta FJ. Tratamiento farmacológico de la dependencia de la nicotina: revisión de la evidencia científica y recomendaciones de la Sociedad Española de Especialistas en Tabaquismo sobre su elección e individualización del tratamiento. Med Clin (Barc). 2011; 136: 79-83.

12. Manual de Abordaje del Tabaquismo en Atención Primaria. Santander: Consejería de Sanidad, Gobierno de Cantabria; 2010

13. SNRT Subcommittee on Biochemical Verification. Biochemical verification of tobacco use and cessation. Nicotine Tob Res. 2002; 4:149-159.

14. Barrueco M, Jiménez C, Palomo L, Torrecilla M, Romero P, Riesco JA. Veracidad de la respuesta de los fumadores sobre su abstinencia en las consultas de deshabituación tabáquica. Arch Bronconeumol. 2005; 41:135-140.

15. Jarvis MJ, Tunstall-Pedoe H, Feyerabend C, Vesey C, Saloojee Y. Comparison of tests used to distinguish smokers from non-smokers. Am J Public Health. 1987; 77: 1435-1438.

16. Carreras JM, Fletes I, Quesada M, Sánchez-Torrecilla I, Sánchez-Agudo L. Diseño y primera evaluación de un programa de tratamiento de tabaquismo por teléfono. Comparación con un modelo estándar. Med Clin (Barc). 2007; 128:247-250.

17. NICE public health guidance 10. Smoking cessation services in primary care, pharmacies, local authorities and workplaces, particularly for manual working 
groups, pregnant women and hard to reach communities. Manchester: National Institute for Health and Clinical Excellence: 2008.

18. Ochoa-Prieto JA, Aurrecoechea Corral R, Llanderas López P Aparicio García S. Tratamiento grupal multicomponente para dejar de fumar en atención primaria. Resultados de 5 años de intervención en un centro de salud. Semergen. 2010; 36: 377-385.

19. Camaralles F, Asensio A, Jiménez Ruiz C, Becerril B, Rodero D, Vidaller O. Efectividad de la intervención grupal para la deshabituación tabáquica. Ensayo clínico aleatorizado. Med Clin (Barc). 2002; 119: 53-57.

20. R'Kaina C, de la Rosa L. Mujer y tabaco: situación actual; enfoque de género. En: Ayesta FJ et al. (eds) Manual de Tabaquismo para profesionales sanitarios. Ceuta: Ingesa-PIT; 2006.p.235:252.

21. Santiago A, Herrán A, Vázquez-Barquero JL. Epidemiología de la enfermedad mental en la comunidad. En Psiquiatría en Atención Primaria. Madrid: Grupo Aula Médica; 1998. p. 35-53.

22. Asthon M, Miller CL, Bowden JA, Bertossa S. People with mental illness can tackle tobacco. Aust N Z J Psychiatry. 2010; 44: 1021-1028.

23. Banham L, Gilbody S. Smoking cessation in severe mental illness. Addiction. 2010; 105: 1176-1189.

24. Hitsman B, Moss TG, Montoya I, George T. Treatment of tobacco dependence in mental health and addictive disorders. Can J. Psychiatry. 2009; 54: 368378.

25. Piper ME, Smith SS, Schlam TR, Fleming MF, Brittich AA, Brown JL et al. Psychiatric disorders in smokers seeking treatment for smoking dependence: relations with tobacco dependence and cesaation. J Consult Clin Psychol. 2010; 78: 13-23. 